\title{
The Impact of Electronic Medical Records on the Work Process of Outpatient Care: Extracting Use-Cases of Paper-Based Medical Records Using a Time Process Study
}

\author{
Sachiko Shimizu ${ }^{1}$, Yuko Ohno ${ }^{1}$, Hiroko Noda ${ }^{1}$, Shohei Nakamura ${ }^{2}$, Ichiro Kanaya ${ }^{2}$, \\ Kenji Yamada ${ }^{1}$, Atsue Ishii ${ }^{1}$, Satoko Kasahara ${ }^{3}$, Katsumi Hirakawa ${ }^{4}$, \\ Rie Nakagawa ${ }^{5}$, and Yasushi Matsumura ${ }^{5}$ \\ ${ }^{1}$ School of Medicine, Osaka University, 1-7Yamadaoka, Suita, Osaka, Japan \\ ${ }^{2}$ School of Engeneering, Osaka University, 2-1Yamadaoka, Suita, Osaka, Japan \\ ${ }^{3}$ School of Medicine, Kochi University, Kohasu Oko, Nankoku, Kochi, Japan \\ ${ }^{4}$ School of Human Nursing, University of Shiga Prefecture, 2500Yasaka, Shiga, Japan \\ ${ }^{5}$ Osaka University Hospital, 2-15Yamadaoka, Suita, Osaka, Japan \\ \{shimizu, ohno, h.noda, atsue\} @sahs.med.osaka-u.ac.jp, \\ \{nakamura, kanaya\} adesign.frc.eng.osaka-u.ac.jp, \\ dr.kenji.yamada@gmail.com, s-kasahara@kochi-u.ac.jp, \\ khirakawa@nurse.usp.ac.jp, \\ \{rnakagawa, matumura\}@hp-info.med.osaka-u.ac.jp
}

\begin{abstract}
The goal of this study is to measure the impact of electronic medical records on both time efficiency and the work process with regard to outpatient care. In this study, we focus on examining the pre-assessment ahead of the introduction of the electronic medical records procedure applying new methodology, time process study. We extracted 12 use-cases and 82 actions in relation to paper based medical records at an outpatient department at a university hospital using time process study. The results suggest that, for nurses, indirect management of patients accounted for a higher proportion of the use-cases than was the case for direct care.
\end{abstract}

Keywords: business intelligence, electronic health records, work process, time efficiency, time process study.

\section{Introduction}

Electronic Medical Records system (EMRs) are intended to improve the efficiency and effectiveness of care through providing assistance to the workload of medical staff. Previous researches in relation to the impact of EMRs have concentrated on assessing the improvement of time efficiency occurring between pre- and postintroduction of EMRs. The crucial question arises as to the ways in which clinical work activities might be subject to change following the introduction of EMRs, and whether the corresponding changes to work activities will affect clinical efficiency. 
The goal of this study is to measure the impact of EMRs on both time efficiency and the work process with regard to outpatient care, with the focus being on examining the pre-assessment ahead of the introduction of the EMRs procedure applying time process study method. The study was undertaken at the outpatient department of Osaka University Hospital, a tertiary care facility located in Osaka, Japan.

\section{Time Process Study}

The Time Process Study (TPS) provided a new method for collecting data as well as for analyzing and visualizing work activity that compensates for the weaknesses of more conventional methodologies. TPS works by utilizing a unified modelling language (UML) to identify and visualize how a business procedure functions.

We suggested extending TPS in the same way as was proposed by Shiki et al. Thus, we first defined the use-case properties in order to identify the characteristics of the outpatient service in relation to paper based medical records (pMRs). In this regard, it is essential both to clarify the process and define the service properties in order that service quality might benefit in terms of improvement.

\section{Results and Discussion}

In this study we extracted 12 use-cases and 82 actions in relation to pMRs at an outpatient department at a university hospital using TPS. The results suggest that, for nurses, indirect management of patients accounted for a higher proportion of the usecases than was the case for direct care. Although some of the tasks a nurse is concerned with may be similar to that of a medical clerk, nursing involves a further role providing direct patient care.

Generally, nursing consists of two roles, namely, a provider of direct patient care and a manager of the care environment. While direct care is an important component of nursing, indirect assistance with regard to care and the medical environment is crucial to the success of any intervention which also applies to all health care providers. Conversely, previous research has shown that the demands related to documentation can have a negative impact on job satisfaction. Our results showed paramedics in outpatient department provide their service to internal customer primary. According to these findings, indirect care seems to place a higher burden on the nurse than was previously thought.

This study also suggests that the items 'Searching pMRs' and 'Delivering pMRs' involve a great many actions due to the complexity of carrying out these tasks. These tasks have possibilities to involve many 'decisions'. Thus, paramedics may often be required to make informed guesses using incomplete information regarding patients due to the fact that many links occur in the process of associating with other sections of the hospital. Moreover, the tasks 'Searching pMRs' and 'Delivering pMRs' can crop up abruptly and thus lead to a disruption of more immediate task demands.

TPS was allowed to discuss about amount of work process and task properties. Future research is required to examine the validity of the TPS and describe activity diagrams of outpatient services, and further research is needed to examine the impact of EMR on time efficiency and work activities. 